\title{
On the largest convex subsets in Minkowski sums
}

\author{
Hans Raj Tiwary* \\ hansraj@kam.mff.cuni.cz
}

Given two point sets $A, B \subset \mathbb{R}^{2}$ of $n$ points each, the Minkowski sum $A+B$ has a quadratic number of points in general. How large can a subset $S \subseteq A+B$ be if $S$ is required to be convex? We prove that when $A$ and $B$ are both convex then $S$ can have only $O(n \log n)$ points. This complements the existing results that are known when $A$ and $B$ are not in convex position or when $B=A$ and $A$ is convex.

\section{Introduction and Related Work}

Let $A, B$ be two sets of $n$ points each in $\mathbb{R}^{2}$. The Minkowski sum $A+B$ is defined as the set of sums obtained from any point in $A$ with any other point in $B$. That is,

$$
A+B=\{a+b \mid a \in A, b \in B\}
$$

Clearly, the size of $A+B$ will be quadratic in general. We call a point set $S$ convexly independent if any of the points cannot be represented as a convex combination of the other points in $S$. Suppose we want to pick a subset of points from $A+B$ such that the points are in convex position, that is, convexly independent. How large can such a subset be picked? Halman, Onn, and Rothblum [3] raised this question in connection with a class of convex combinatorial optimization problems [4].

Note that when the sets $A$ and $B$ are allowed to be arbitrary then one can just look at the case when $A$ and $B$ are the same set. Simply consider the set $X=A \cup B$. Any asymptotic bound for convex subsets of $X+X$ then applies for convex subsets of $A+B$ and vice versa. In fact, Halman et al. [3] asked the question for the case when the two sets are the same. They proved that for a convex set $X$ of points in the plane at most $5 n-6$ points of $X+X$ can be in convex position. They asked whether for general point sets a subquadratic upper bound exists. Note, however, that for convex point sets the case when $A=B$ is distinct from the case when $A \neq B$.

Eisenbrand et al. [2] answered the question of Halman et al. in affirmative by showing that for general point sets, no convex subset of the Minkowski sum can be larger than $O\left(n^{\frac{4}{3}}\right)$. Later Buchin et al. [1] showed that for general point sets this bound was optimal by giving a construction where a subset of size $\Omega\left(n^{\frac{4}{3}}\right)$ can be picked from the Minkowski sum of two point sets of size $n$.

\footnotetext{
${ }^{*}$ Department of Applied Mathematics (KAM) and Institute of Theoretical Computer Science (ITI), Charles University, Malostranské nám. 25, 11800 Praha 1, Czech Republic
} 
Therefore, for general point sets the question has been completely answered. However, as proved by Halman et al. for convex point sets this bound is never achieved. We look at the case when the two sets $A, B$ are both in convex position but not necessarily the same. As remarked earlier, this case is distinct from the case when $A$ and $B$ are the same convex point sets. For the case when $A$ and $B$ are convex but not necessarily the same, we prove the following:

Theorem 1. Let $A$ and $B$ be two planar convex point sets with $|A|=m,|B|=n$. For any convexly independent subset $S \subseteq A+B$, we have $|S|=O((m+n) \log (m+n))$.

The original motivation of Halman et al. [3] for studying this problem arises from the notion of the convex dimension of a graph. The convex dimension of a graph is defined as the smallest dimension in which the graph can be embedded such that midpoints of all the edges lie in convex position. The strong convex dimension of a graph is defined to be the smallest dimension in which the graph can be embedded such that the vertices are in convex position as well as the midpoints of all the edges are in convex position.

Haltman et al. were interested in studying the extremal properties, such as the maximum number of edges, of graphs that have convex dimension (strong or otherwise) two. Their result was a linear upper bound on the number of edges of graphs with strong convex dimension two, whereas the result of Eisenbrand et al. gives an upper bound on the number of edges of a graph whose convex dimension is two.

Our result does not fit immediately into the framework of convex dimensions as such, but one can possibly generalize the notion of convex dimension in the following way. Instead of requiring that either all the vertices lie in convex position in the embedding (the case of strong convex dimension, or equivalently the case of the Minkowski sum of a convex set with itself) or dropping this requirement altogether (the case of convex dimension or the Minkowski sum of a general point set with itself), one can require that the embedding be such that the point set can be decomposed into $k$ convex subsets, with $k$ some parameter. Our result would imply an upper bound of $O(n \log n)$ for the case $k=2$.

\section{Proof of Theorem 1}

A point set in $\mathbb{R}^{2}$ in convex position is said to define a monotone chain if both the $x$ and the $y$ coordinates are monotone. It is easy to see that any point set in convex position can be decomposed into at most four subsets such that each subset defines a monotone chain. See figure 1 for example.

Therefore, we can consider each of the convex chains separately and bound the size of a convexly independent subset from each pair of these chains from $A$ and $B$.

Observation 1. For any two point sets $A, B$, translating either set by a fixed vector does not change the number of points of the Minkowski sum $A+B$ that are convexly independent.

To see this note that upon adding a vector $c$ to every point in, say, set $A$ every point $p$ of the Minkowski sum $A+B$ moves to $p+c$. Thus, whether a subset is convexly independent or not is not affected.

A monotone chain can be one of four types depending on whether the $x$ and the $y$ coordinates are non-decreasing or non-increasing. If we consider two convex chains of different types then it is easy to see that one of the sets can be translated so that all points of both the sets taken together still lie in convex position. It follows from the result of [3] that in this case the largest convexly independent subset of the Minkowski sum of the two sets can not be more than 


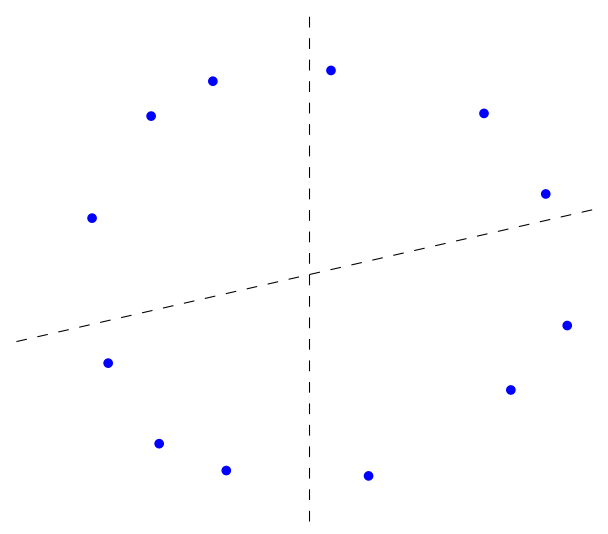

Figure 1: Each quadrant contains a subset of points lying on a monotone chain.

$O\left(m_{i}+n_{j}\right)$, where $m_{i}$ is the size of the monotone chain from $A$ of $i$-th type and $n_{j}$ is the size of the monotone chain from $B$ of $j$-th type. Hence, the total size of a convexly independent subset of $A+B$ that arises only from the sums of points in $A$ and $B$ that lie on chains of different type is bounded by $O(m+n)$.

Now, consider the pairing of same type of monotone chains from $A$ and $B$ of lengths $m_{i}$ and $n_{i}$ respectively. Without loss of generality, we can assume that both the chains have $x$ and $y$ coordinates non-decreasing. Consider the $m_{i}+n_{i}-2$ different slopes in each of these two chains and consider the median slope. Let $p_{a}$ be the right end point of the segment in $A$ and $p_{b}$ be the right end point of the segment in $B$ whose slopes are the largest in their chains below the median slope (See Figure 2).
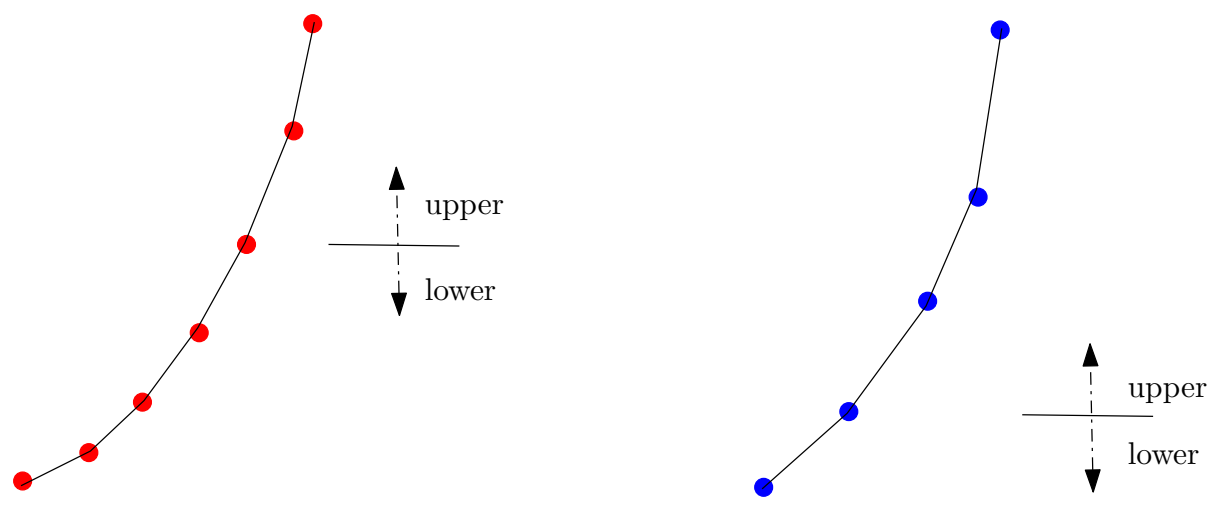

Figure 2: Partition of each chain into an upper and a lower subchain wrt the median slope.

These points break each chain into a lower and an upper subchain. It is clear that the lower subchain from one set can be translated so that together with the upper subchain of the other set it produces a convex chain. Therefore, we can have only a linear number of convexly independent points from these pairings.

The lower (reps. upper) subchain of one can however still produce more points from the interaction with the lower (upper resp.) subchain of the other chain. Let $f(m, n)$ count the maximum size of any convexly independent subset of two convex point sets of similar type with 
number of edges in the respective chains $m$ and $n$, then we get the following recursion:

$$
f(m, n) \leq O(m+n)+f\left(m_{u}, n_{u}\right)+f\left(m_{b}, n_{b}\right),
$$

where $m_{u}+n_{u} \leq\left\lfloor\frac{m+n}{2}\right\rfloor$, and $m_{b}+n_{b} \leq\left\lceil\frac{m+n}{2}\right\rceil$. Therefore,

$$
f(m, n)=O((m+n) \log (m+n)) .
$$

Since subchains of similar type can produce only a linear number of convexly independent subsets in the Minkowski sum the bound on $f(m, n)$ applies for the entire point set $A$ and $B$. This concludes the proof of Theorem 1.

\section{Concluding Remarks}

The proof of Theorem 1 seems to be very generous with counting which points of the Minkowski sum may be selected to be convexly independent. We do not know whether this is really the case and the upper bound can be improved by more careful argument. The only lower bound that we are aware of, for the specific case discussed here, is the trivial linear bound. We leave this disparity between the upper and the lower bounds as an open problem.

\section{References}

[1] K. Buchin, R. Fulek, M. Kiyomi, Y. Okamoto, S. Tanigawa, and C. D. Tóth. A tight lower bound for convexly independent subsets of the minkowski sums of planar point sets. In Proc. 7th Japan Conference on Computational Geometry and Graphs, 2009.

[2] F. Eisenbrand, J. Pach, T. Rothvoß, and N. B. Sopher. Convexly independent subsets of the Minkowski sum of planar point sets. Electronic Journal of Combinatorics, 15(1):Note 8, 4, 2008. Professor Pach's number: [224].

[3] N. Halman, S. Onn, and U. G. Rothblum. The convex dimension of a graph. Discrete Applied Mathematics, 155(11):1373-1383, 2007.

[4] S. Onn and U. G. Rothblum. Convex combinatorial optimization. Discrete \& Computational Geometry, 32(4):549-566, 2004. 